\title{
Cross-modal decoupling in temporal attention between audition and touch
}

\author{
Stefanie Mühlberg ${ }^{1}$ and Salvador Soto-Faraco ${ }^{1,2}$ \\ 1 Center of Brain and Cognition, Universitat Pompeu Fabra, Barcelona 08018, Spain \\ 2 ICREA, Institució Catalana de Recerca i Estudis Avançats, Barcelona 08010, Spain
}

Keywords: temporal attention, behaviour, cross-modal, audition, touch

Corresponding Author: Stefanie Mühlberg

Affiliation:

Center for Brain and Cognition (CBC), Universitat Pompeu Fabra, Spain

Email:

stefanie.muehlberg@upf.edu

Telefone:

+34935422534

ORCID:

Stefanie Mühlberg - http://orcid.org/0000-0003-2848-6367

Salvador Soto-Faraco - http://orcid.org/0000-0002-4799-3762

Postal address:

Departament de Tecnologies de la Informació i les Comunicacions

Universitat Pompeu Fabra

Carrer de Ramon Trias Fargas, 25-27

Edifici Mercè Rodoreda (Room 24.327)

08005 Barcelona

Spain

Fax number: $\quad$ +34935422517

Word count:

Total

10953

Abstract

200 


\section{Introduction}

Attention allows one to segregate relevant from irrelevant information (e.g., Treue, 2003), to allocate resources accordingly, and to improve their processing. One of the possible dimensions in which attention can be directed is time. Temporal attention leads to processing improvements for events occurring at attended moments, leading to faster responses (e.g. Correa et al., 2004; Coull \& Nobre, 1998; Griffin et al., 2001; Miniussi et al., 1999), increased accuracy (Correa \& Nobre, 2008) and lower perception thresholds (Cravo et al., 2013; Rohenkohl et al., 2012). Physiologically, temporal attention has been found to modulate early event-related potentials (ERPs) such as the N100 component (Lange et al., 2003; Lange et al., 2006; Lange, 2012; Miniussi et al., 1999; Sanders \& Astheimer, 2008), as well as later components such as the P300 (Lampar \& Lange, 2011; Miniussi et al., 1999) and the N200 (Sanders \& Astheimer, 2008).

Although temporal attention has been mostly studied in unisensory contexts, everyday life environments are multisensory by nature; thus, the brain must constantly face the problem of selecting information at the relevant times as well as in the relevant modality (ten Oever et al., 2016). A classic question that arises from this selection problem is: Does attentional orienting in one dimension affect the processing of all possible modalities (e.g. a supramodal attention system), or is attentional orienting managed independently for each sense? (e. g. Driver \& Spence, 1998a, 1998b; Lange \& Röder, 2006; Spence \& Driver, 1997). This question regarding cross-modal selective attention has mostly been researched in the domain of spatial attention, and a general agreement has emerged over the last 20 years that orienting attention to a spatial location in one sensory modality induces corresponding shifts in other sensory modalities. This leads to behavioural benefits at the attended location for all possible modalities. This pattern could be described as cross-modal spatial coupling (e.g. Driver \& Spence, 1994; Eimer \& Driver, 2000; Eimer et al., 2002; Eimer, 1999; Macaluso \& Driver, 2005; Macaluso et al., 2000; Macaluso, 2010; Sambo \& Forster, 2011; Santangelo et al., 2010; Spence \& Driver, 1997, 1996; Spence et al., 2000; Tang et al., 2015; Trenner et al., 2008). However, in contrast to spatial attention, the consequences of cross-modal orienting in time have been far less studied and the interpretation of the results is still controversial (Lange \& Röder, 2006; Mühlberg et al., 2014).

Lange and Röder (2006) conducted a relevant study that addresses the question of orienting temporal attention in a cross-modal environment. Their paradigm involved a discrimination task (single vs. double event judgment) on targets that could be either auditory or tactile, and presented at an early or late time point after a 
warning cue. Based on previous endogenous spatial attention studies in cross-modal contexts (Spence \& Driver, 1996, 1997), target modality and time were manipulated probabilistically so that that the majority of all targets presented at the expected time point belonged to the primary modality. Instead, most targets presented at the unexpected time point belonged to the secondary, less frequent, modality. Hence, Lange and Röder's experiment design (followed by Mühlberg et al.'s 2014, and the present study) used a probabilistic manipulation, with the general assumption that manipulations of expectancy can induce corresponding orienting of top-down attention (see Correa et al., 2005; Nobre \& Rohenkohl, 2014). Attention and expectation are technically dissociable processes, with different expressions in terms of brain correlates (Kok et al., 2012; Summerfield \& Egner, 2009), but have hardly distinguishable behavioural outcomes. In line with Summerfield and Egner (2009), we can consider that expectation can be modulated via likelihoods (the variable directly manipulated here), whereas attention relates to motivational relevance (which can be based, amongst other things, on known likelihoods). That is, the more likely a target is to appear (expectation manipulation), the more behaviourally relevant it becomes (attention manipulation). To further support this motivational relevance, the probabilistic manipulation can be supported by explicit instructions. This is in order to encourage attention shifts towards the more likely time/modality of the upcoming target (e.g., Lange \& Röder 2006). An additional separation has to be made between time-based and temporal attention. Time-based attention refers to the anticipation, and potentially orientation of attention, towards an event coupled to a temporal marker (Aufschnaiter et al. 2017; Thomaschke \& Dreisbach, 2015; Thomaschke et al., 2015; Thomaschke et al., 2011; Volberg \& Thomaschke, 2017; Wagener \& Hoffmann, 2010). In other words, time is informative about a target property, such as color or modality. In contrast, temporal attention itself would refer to focusing upon a certain point in time (Los et al., 2001; Schröter et al., 2015; Thomaschke \& Dreisbach, 2015; Thomaschke et al., 2015). The case addressed here, just like Lange and Röder 2006, and Mühlberg et al., 2014, deploys both types of orienting in time, since the likelihood of the target modality is contingent upon time points (time-based attention), yet, the participants' orientation of attention in time is determined by the overall probability of target appearance (temporal attention). Following this logic, one could formulate the original question of this paper in terms of the interplay between time-based attention (to modality) and temporal attention (e.g., attention to time).

In Lange and Röder's (2006) study, the results showed a speed up in reaction times and a modulation of the N100 ERP component for targets in both modalities (primary and secondary) at the overall most likely 
time interval. This pattern suggested a cross-modal coupling in the allocation of temporal attention, similar to previous results in cross-modal spatial attention (e.g., Spence \& Driver, 1996). However, Lange and Röder were only able to analyse the responses provided at short time intervals because the experimental protocol did not induce uncertainty about target presence at the later time point (Coull \& Nobre, 1998; Griffin et al., 2001). In order to investigate the responses at both time points, Mühlberg et al (2014) adjusted a paradigm previously used by Spence and Driver (1996), where two modalities, vision and touch, could be presented at two possible onset points, early and late, but with different temporal likelihoods. Mühlberg's study introduced catch trials without stimuli were used to prevent certainty at the late time point. In contrast to the results of Lange and Röder (2006), the results of Mühlberg et al. (2014) showed that each modality could benefit from its relative appearance probabilities at different points in time. In other words, the pattern was consistent with cross-modal decoupling in temporal attention. The findings are in stark contrast to Lange and Röder's (2006), yet the source of this difference remained unclear.

One obvious possibility is that the disparity between the results of Lange and Röder (2006) and Mühlberg et al. (2014) simply reflects that different sensory modality combinations are subject to varying degrees of coupling/decoupling. In this case, touch and audition would be subject to a stronger coupling than touch and vision when it comes to orienting attention to (and/or) expecting events in time. Similar modality asymmetries have been reported before, for example, in the case of cross-modal spatial attention (Klein, 1977; Posner et al., 1976), leading to rare cases of a spatial cross-modal decoupling in space as well. For example, Lloyd et al's study (2003) shows partial evidence of cross-modal decoupling in spatial attention between audition and touch, the same modality pairing as Lange and Röder (2006) and the present study. In Lloyd et al.'s study, the authors found that participants were capable of monitoring different modalities at different locations, but at the expense of an overall performance decrease, which would still align with the cross-modal synergy proposed by Spence \& Driver (1996; see also Soto-Faraco et al., 2005, for another failure to find crossmodal synergies in an audio-visual sustained spatial attention task). Perhaps, because audition and touch are less spatially dominant than vision (Welch \& Warren, 1980), spatial coupling is easier to overcome between these two modalities. In contrast to that, temporal information vision is less reliable than audition and touch (Kubovy, 1988; VanRullen et al., 2014). Following up on these speculations, cross-modal coupling in temporal 
attention may be stronger in the presence of temporally dominant auditory stimuli (as in Lange and Röder, 2006), than for other pairings such as vision and touch (as in Mühlberg et al., 2014).

However, there may be a different possibility to explain previously discrepant results: temporal orienting of attention is fundamentally different from spatial orienting of attention in terms of how strongly different sensory modalities follow each other. Here, we argue that there is a basic difference between temporal and spatial attentional orienting that could possibly lead to a difference in their behavioural expression in crossmodal contexts (see Mühlberg et al. 2014). Spatial attentional orienting usually involves the simultaneous processing of (or preparation for) alternative events, which compete for resources within the same time window (Desimone \& Duncan, 1995). In contrast, in temporal selective attention, orienting occurs serially by definition; therefore, allowing a flexible allocation of processing resources at various relevant moments in time (Mühlberg et al., 2014). This tentative hypothesis is indirectly supported by differences in neural correlates between spatial (Eimer \& Driver, 2000; Störmer et al., 2009; Yang \& Mayer, 2013) and temporal attention (Coull \& Nobre, 1998; Davranche et al., 2011; Lampar \& Lange, 2011; Miniussi et al., 1999; Sanders \& Astheimer, 2008) in ERP and fMRI studies. Along the same line, a recent EEG study by Keil et al. (2017) suggested that temporal and modality attention operate with different latencies and brain sources. If temporal and spatial attention are indeed intrinsically different, then cross-modal decoupling might represent a general property of temporal attention, at variance with the cross-modal coupling usually found in spatial attention. Therefore, one should be able to find evidence for decoupling between other pairs of modalities including audition and touch, just like Mühlberg et al. (2014) had previously found with touch and vision.

To test whether decoupling can emerge between audition and touch, we followed the same variation of Lange and Röders's paradigm (2006) used in Mühlberg et al. (2014), with the sole adjustment of using auditory and tactile events. The probability of target stimuli in one of the two modalities and at one of two possible onset times was higher (primary modality, at the expected time point). Importantly, the relative probability of target modality in the less likely time point was reversed. This design allows a direct comparison of the results of Lange and Röder (2006) and Mühlberg et al. (2014). We hypothesize here that, if cross-modal temporal decoupling occurs, based on the specific temporal likelihoods of auditory and tactile stimuli, then we should observe that both modalities show the best behavioural responses at their respectively more likely point in time. In this design, this means that the primary and secondary modalities should display reversed temporal 
cueing patterns. However, if cross-modal temporal attention is coupled for touch and audition, then both the primary and secondary modality targets should show better performances at the overall most likely point in time. In the present design, this means that the primary and secondary modalities should display parallel temporal cueing patterns. Reaction times and accuracy were used as a proxy for target processing efficiency.

\section{Experiment 1}

\section{Methods}

\section{Participants}

A total of 29 participants volunteered for this experiment ( 2 left-handed; 20 female; mean age 25.92 years, age-range 18-61 years) in exchange for $8 €$ per hour. They all reported normal or corrected-to-normal vision.

\section{Stimuli}

The stimuli used as targets in the study were auditory or tactile, and presented as single or double pulse events. Auditory stimuli consisted of short auditory tones $(1000 \mathrm{~Hz}, 60 \mathrm{~dB}(\mathrm{~A}))$ delivered via headphones at a comfortable loudness, presented to the left or the right ear. Single pulse stimuli lasted 50ms, whereas double pulse stimuli consisted of two pure tone bursts of $10 \mathrm{~ms}$ duration, separated by a $30 \mathrm{~ms}$ gap. Tactile stimulation was presented on the tip of the left or the right index finger of the participant's hand and was delivered by a solenoid tapper (round tip, 8 mm, Miniature Solenoid Tapper Controller Mk3, MSTC3-10M, M\&E Solve, UK). For single pulse stimulation, the tapper was lifted for $10 \mathrm{~ms}$; double pulse stimuli consisted of two $2 \mathrm{~ms}$ stimulations, separated by an $8 \mathrm{~ms}$ gap. The tactile stimulation did not cause any pain or annoyance to the participant. These parameters of the auditory and tactile stimuli were adjusted based on prior informal pilot testing, so that the accuracy for both would exceed chance level.

\section{Experimental design and procedure}

The experiment protocol and response collection protocols were custom programmed using MatLab 8.3.0 (The MathWorks Inc.; Natick, MA, USA). The goal of this study was to investigate if cross-modal decoupling in time (Mühlberg et al., 2014) extends to a different set of modalities such as audition and touch. In order to 
do so, we adjusted the task design from Mühlberg et al. (2014) to meet the needs of the aforementioned experimental purpose.

Participants performed a one vs. two pulse discrimination task over tactile and auditory stimuli in a paradigm where we manipulated the participants' expectations about the onset time and modality of the upcoming target. The experiment was conducted in a sound attenuated room with dim illumination. Participants sat in a chair with their arms relaxed on each side, on the chair armrests. A monitor placed in front of the participants displayed a black fixation cross on a grey background and two grey arrows on the left and right side of the fixation cross, respectively pointing left and right. In order to start a trial, participants had to simultaneously press two foot pedals, placed underneath the heel and the toes of their dominant foot, and they remained pressing the pedals at all times until they until they responded. If participants failed to do so, then the trial was discarded and repeated at a later time within the block. Each trial started with a colour change of one of the arrows from grey to black, which indicated the side of the upcoming stimulus with $100 \%$ validity. A target stimulus could appear which were either auditory or tactile, single or double pulse stimulation, 1.2s (early) or 2.4s (late) after this spatial cue. Participants had to discriminate if the target stimulus had been a single or double. In order to do so, participants had to release one of the two foot-pedals (heel or toe, response-mapping counterbalanced across participants). Participants were informed before every block about the most likely time-point of target appearance and modality, and instructed to answer as quickly and accurately as possible. After the response (or after the response timeout of 3s), an inter-trial interval of $1 \mathrm{~s}$ led to the beginning of the next trial. Masking white noise was played over the headphones throughout the whole length of the trial (from the cue) in order to block out the slight noise produced by the solenoid tappers.

To manipulate the participants' expectation about onset time and target modality, and hence inducing the orientation of endogenous attention in time and modality, we adjusted the likelihoods of these two factors in a probabilistic manner (Fig. 1). Temporal expectation about onset time was manipulated in a block wise manner. Targets would appear at the expected onset time in $54 \%$ of the trials, compared to only $23 \%$ of trials containing the target at the unexpected onset time. The remaining $23 \%$ of trials were catch trials where no target would 
appear at all. Note that every trial contained a maximum of one target, so that auditory and tactile targets could not be presented in the same trial.

To manipulate modality prevalence, we made one of the two target modalities, called primary modality, more likely to appear than the other, accounting for a total of $70 \%$ of all targets; whereas, only $30 \%$ of all targets belonged to the other, secondary modality. Whether the modality would be the primary modality or the secondary modality was fixed for each participant and encoded in the factor primary modality. Since one of the participants was excluded from the analysis due to low accuracy (chance performance) in one of the experimental conditions, this resulted in a total of 28 participants in the analysis. The factor of primary modality was counterbalanced between participants and each of the groups contained a total of 14 participants.

Most importantly, in order to measure any decoupling between modality and time, the factors of modality prevalence and temporal expectation were dissociated from each other in the following way. At the expected time point, the majority of targets $(86 \%)$ were primary modality targets and only the remaining $14 \%$ of targets were in the secondary modality. At the unexpected time point, the likelihoods reversed, so that $67 \%$ of targets were in the secondary modality and the remaining $33 \%$ were in the primary modality.

Every participant ran a total of six experimental blocks of 108 trials each (a total of 648 trials). Within three of the experimental blocks, the early onset would be the expected onset $(1.2 \mathrm{~s})$. Within the remaining three blocks, the late onset $(2.4 \mathrm{~s})$, was presented in the order of conditions, AAABBB, counterbalanced between participants. One experimental session lasted approximately 45mins. During the experiment, reaction times and response accuracy were recorded. Trials in which the participants failed to provide a response or in which the foot pedals were released before the response window were automatically discarded and repeated at the end of the block.

In order to learn the probabilities of the different experimental conditions, participants performed the task on a training set of 36 trials. The training only consisted of half of the primary targets at the expected onset and half of the secondary targets at the unexpected onset of the first three experimental blocks. To learn the temporal 
properties, further visual temporal information was given throughout the training. After the initial cue, the arrow corresponding to the cued side would flicker every $400 \mathrm{~ms}$ for $50 \mathrm{~ms}$, resulting in two flickers before an early target and five flickers before a late target. Participants were made aware of this supporting temporal information and instructed to use it. Importantly, this additional temporal information was not presented during the actual experiment. Additionally, a second training set, consisting of 24 trials, was presented after the first three blocks in order to enable the participant to adapt to the new temporal expectation pattern for the next three blocks. The data from the training sets were not analysed.

Analysis

Similar to Mühlberg et al (2014), all incorrect responses and RTs that were two standard deviations away from the individual mean were discarded from the analysis ( $<5 \%$ of all trials were excluded). Furthermore, the analysis focused on mean reaction times (RT) and accuracy. ${ }^{1}$

In order to analyse the interaction of attention to time and modality, we ran an ANOVA with the betweengroup factor of primary modality (audition or touch), within-group factors of modality prevalence (primary or secondary), onset time (early or late) and block type (expect early or expect late). A significant interaction between attention to time and modality would thereby be reflected through a three-way interaction between modality prevalence, onset time and block type. Fisher LSD tests were used as a post-hoc analysis. All statistical analyses were conducted with STATISTICA 8.0 (StatSoft Inc.; Tulsa, OK, USA). Note that any possible asymmetry between primary modalities would be reflected in the four-way interaction, involving primary modality.

\footnotetext{
${ }^{1}$ In addition to mean reaction times and accuracy, we analysed the obtained median reaction times and inverse efficiency. However, since the results for the median and mean reaction times were in accordance to each other, and since no trade-offs between reaction times and accuracy could be found, we decided to only report the mean reaction times, since they have also been previously reported in Mühlberg et al. (2014), as well as in Lange and Röder (2006).
} 


\section{Results}

Mean RTs

We found a significant main effect of modality prevalence $\left(\mathrm{F}_{1,24}=22.25, \mathrm{p}<0.01\right)$, in which participants responded faster towards primary modality targets than secondary modality targets. Furthermore, this effect was modulated by the primary modality (interaction modality prevalence $\mathrm{x}$ primary modality; $\mathrm{F}_{1,24}=12.54$, $\mathrm{p}<0.01)$. This shows that the main effect of modality prevalence was mostly driven by the audition group, where we found a large difference between the primary (audition, $807 \mathrm{~ms}$ ) and the secondary modality (touch, 992 ms; $\mathrm{p}<0.01$ ); whereas, there was no significant difference between the responses towards primary (touch) and secondary (auditory) modality targets within the touch group ( $878 \mathrm{~ms}$ vs. $904 \mathrm{~ms}$, respectively; $\mathrm{p}=0.41$ ). No other main effect reached significance. However, we found two close-to-significant interactions: one between modality prevalence, onset time, and block type $\left(\mathrm{F}_{1,24}=3.89, \mathrm{p}=0.060\right.$, Fig. $\left.2 \mathrm{~A}\right)$, and one between modality prevalence, onset time, block type, and the primary modality $\left(\mathrm{F}_{1,24}=3.85, \mathrm{p}=0.061\right.$, Fig. $\left.2 \mathrm{~B} \& \mathrm{C}\right)$. This nearsignificant interaction is relevant in terms of one of our initial hypotheses: namely, that different modalities possess different temporal reliabilities and thus, might be prone to different levels of cross-modal coupling. To test this hypothesis, we decided to follow up the four-way interaction between the primary modality, modality prevalence, onset time, and block type. In the touch group, the pattern of RTs followed the relative likelihoods of target appearance in each modality, meaning that participants showed a significant $(\mathrm{p}=0.046$, expect early blocks, Fig. 2B) or nearly significant ( $\mathrm{p}=0.068$, expect late blocks, Fig. 2B) advantage for the primary modality targets at the expected time points. Meanwhile, the secondary modality targets showed no such trend $(\mathrm{p}=0.92$ for expect early blocks and, $\mathrm{p}=0.15$ for expect late blocks, Fig. 2B). In the audition group, neither the primary modality (audition, $\mathrm{p}=0.53$ for expect early and $\mathrm{p}=0.90$ for expect late blocks, Fig. $2 \mathrm{C}$ ), nor the secondary modality (touch, $\mathrm{p}=0.95$ for expect early and $\mathrm{p}=0.67$ for expect late blocks, Fig. $2 \mathrm{C}$ ) showed any temporal attention effect. In other words, while the trends within the touch group clearly suggest a decoupling of attention to time and modality, the results of the audition group reflect no temporal modulation at all; hence, these results are uninformative with respect to either coupling or decoupling. 
Accuracy

On average, the accuracy during the experiment was high (92.5\% of correct responses). We found a significant interaction between modality prevalence and primary modality $\left(\mathrm{F}_{1,24}=57.91, \mathrm{p}<0.01\right)$, revealing more accurate responses towards the primary vs. secondary modality for the audition group $(97.6 \%$ vs. $87.5 \%, \mathrm{p}<0.01)$ and more accurate responses towards the secondary vs. primary modality for the touch group (88.3\% vs. $96.4 \%$, $\mathrm{p}<0.01)$. This pattern simply reflects that responses towards auditory targets were overall more accurate than towards tactile targets. This effect was also further modulated by the onset time, leading to a three-way interaction between primary modality, onset time and modality prevalence $\left(\mathrm{F}_{1,24}=7.27, \mathrm{p}=0.013\right)$, showing that the decreased accuracy for tactile targets was particularly strong for the expect early blocks of the audition group ( $\mathrm{p}=0.017)$. Additionally, we found a trend for a main effect of block type $\left(\mathrm{F}_{1,24}=3.55, \mathrm{p}=0.072\right)$, suggesting that participants were slightly more accurate for the expect early blocks than the expect late ones (93.4\% vs. $91.6 \%)$. No other main effect or interaction reached significance.

\section{Discussion}

According to the hypothesis of cross-modal coupling in temporal attention, we would expect this: during the moments when attention is oriented towards one sensory modality, targets in other sensory modalities would also be facilitated (Lange \& Röder, 2006). In experiment 1, we pitched relative time expectancies of two different modalities against each other, and failed to find any evidence for such cross-modal temporal coupling hypothesis. Instead, we found evidence for decoupling (touch group), similar to what occurred with touch and vision (Mühlberg et al., 2014), and a lack of evidence for either coupling or decoupling (auditory group). The decoupling revealed itself as a trend towards an interaction of temporal expectation and modality prevalence. Strong expectations about a tactile target at a particular time point were not expressed as an advantage for auditory targets presented at that time point. Instead, if anything, responses to auditory targets tended to follow their own likelihoods in time.

The failure to find coupling in both groups of experiment 1, as well as the existence of this decoupling pattern in the touch group are sufficient grounds to rule out the generality of the cross-modal coupling hypothesis. Yet, the results of experiment 1 also show a pattern in the auditory group that is clearly different 
from what would be expected by the decoupling hypothesis. Indeed, we found an interaction for temporal expectation and modality prevalence for the touch group, but not for the audition group, which makes us question the generality of the decoupling effect as well. At this point, one possible trivial explanation of the failure of the decoupling pattern to arise in the auditory group would be that the discrimination of the auditory targets was simply too easy. It is clear that auditory target discrimination was easier than the tactile one, leading to significantly faster and more accurate responses to auditory targets compared to tactile targets. This means that selective attention to audition might not have been fully required to respond efficiently to auditory targets, especially in the auditory group (Spitzer et al., 1988). It is conceivable that due to the low auditory task difficulty and auditory temporal dominance (Kubovy, 1988; Welch \& Warren, 1980), the possible temporal effects of the primary (auditory) and secondary (tactile) modalities were reduced when audition was the most frequent modality. Note that, strictly speaking, the coupling hypothesis would predict a strong temporal orienting effect for the primary modality, together with a trend in the same direction for the secondary modality. Instead, temporal orienting failed to occur altogether in this group ${ }^{2}$.

An alternative hypothesis to explain the pattern of results in experiment 1 can be based on the special role of orienting towards or away from touch (Spence et al., 2001). Allocating attention towards or away from touch seems to produce higher costs in the form of slower reaction times compared to visual or auditory stimuli for both attended and unattended tactile stimuli. Thus, attending to (difficult) touch stimuli and away from (easy) auditory stimuli in the touch group might help balance the two modalities and create the need for attentional selection, leading to decoupling. In the audition group, however, participants might instead forgo the need to allocate attention to (already easy) auditory events. Therefore, this may allow them to deal with (difficult) tactile information when it comes.

Another factor that might have influenced the pattern of results in experiment 1 is the absence of spatial alignment for auditory and tactile stimuli. Since the tactile and auditory stimuli in the study of Lange and Röder (2006) were presented in close spatial proximity, this could be a determining difference between these two

\footnotetext{
${ }^{2}$ In other words, like we have a high spatial resolution for vision and therefore visual dominance in crossmodal spatial paradigms (e.g. ventriloquist effect; Jackson, 1953; Slutsky \& Recanzone, 2001; Spence, 2010; Welch \& Warren, 1980), the resolution for audition in the temporal domain is higher than for vision or touch (Bresciani et al., 2005; Fendrich \& Corballis, 2001; Morein-Zamir et al., 2003) and therefore audition would lead in temporal attention paradigms, dominating over all possible modulations in other modalities. (This sentence is too long and I don't know how to cut it, because I might change the meaning).
} 
studies. While technically possible, this explanation alone seems unlikely to account for the overall failure of coupling to occur (and decoupling to arise). The spatial disparity between the location of the tactile and auditory stimuli in experiment 1 was restricted only to a few degrees in depth and elevation within the same hemifield, and some evidence suggests that audio-tactile stimulation can lead to similar neural correlates and behavioural interactions even in spite of approximate or a lack of spatial alignment (e.g., Murray et al. 2005). Additionally, Lloyd et al. (2003) investigated the cross-modal spatial interplay of attention between audition and touch and found the same pattern of results for spatially congruent and incongruent auditory and tactile events. Given these precedents, we believe that the current differences in spatial location should be inconsequential to the interpretation of the result, although we cannot rule out the possibility completely.

Altogether, the pattern observed in experiment 1 did not support a cross-modal coupling, but only provides weak evidence for a cross-modal decoupling as described in our initial hypotheses (see also, Mühlberg et al. 2014). One possible reason may simply be a lack of statistical power; however, considering that the number of participants and trials is comparable to Mühlberg et al. (2014), this seems to be an insufficient conclusion. Nevertheless, this question remains to be open to discussion with regards to how far task difficulty and spatial misalignment have influenced the pattern of results in experiment 1 . In experiment 2 , we address these two factors by investigating whether the pattern of results (when audition is primary) becomes clearer when increasing the difficulty of the auditory task and, whether spatial alignment (vs. misalignment) leads to coupling.

\section{Experiment 2}

\section{Methods}

Participants

A total of 28 new participants volunteered for this experiment ( 3 left-handed, 24 female, mean age 23.21 years, age range 18-35 years) in exchange for $8 €$ per hour. They all reported normal or corrected-to-normal vision. 


\section{Stimuli}

The goal of this experiment was to test potential alternative explanations for the findings of experiment 1 . Firstly, we examined whether task difficulty had masked possible coupling/decoupling in the audition group of experiment 1 . Secondly, we tested whether the failure to find coupling in the first experiment might be due to the spatial misalignment of the auditory and tactile targets. In order to address the first issue, we adjusted the loudness of the auditory stimuli in comparison to the background white noise for each individual separately until participants reached an auditory discrimination training performance of approximately $80 \%$. We also enquired whether participants subjectively perceived the discrimination task as equally difficult for both auditory and tactile stimuli, and if they perceived the task as perceptually demanding. To address the second issue, we divided the group of 28 participants in two groups of 14 participants each (that is, each group equalled the $\mathrm{N}$ of the auditory group in experiment 1 ). In the first group (misaligned), we presented the auditory stimuli via headphones, as in experiment 1 . In the second group (aligned), we presented the auditory stimuli via loudspeakers located close to the participants' hands to ensure spatial proximity between auditory and tactile stimuli. All sounds were presented at a comfortable loudness of about $60 \mathrm{~dB}(\mathrm{~A})$, measured from the participants head.

\section{Experimental Design}

The experimental design was mostly identical to that of experiment 1 . However, since the interest of this new experiment referred to the results of the auditory group of experiment 1 , all of our participants had audition as their primary modality and touch as their secondary modality, removing the factor of primary modality from the design.

\section{Analysis}

The analysis was conducted following the structure of experiment 1 , with the additional betweenparticipants factor of spatial alignment and without the between-participants factor of primary modality. Hence, the variables were the within-subject factors of modality prevalence (primary or secondary), onset time (early or late), and block type (expect early or expect late), and the between-subject factor of spatial alignment (aligned or misaligned). Additionally, to test for task difficulty, we ran an ANOVA over the results of the audition group 
in experiment 1 (easy) and the data of the misaligned group of experiment 2 (difficult). We used RT and accuracy as dependent variables (in separate analyses).

\section{Results}

Mean RTs

We ran an ANOVA with the between-group factor of spatial alignment and the within-group factors of modality prevalence, onset time, and block type. None of the main terms of the ANOVA reached significance. However, we found that the critical pattern revealing an interaction between attention to time and to modality, was significant (interaction between modality prevalence, onset time and, block type $F_{1,24}=6.97, p=0.014$, Fig. 3). Looking at this interaction more closely, post-hoc analyses (Fisher LSD) showed non-significant trends towards decoupled attention effects in expect early blocks. That is to say there was an advantage for expected primary modality targets ( 855 vs. $893 \mathrm{~ms}, \mathrm{p}=0.069$, Fig. 3A), and a (numerical) advantage in the opposite direction for secondary modality targets ( 925 vs. $894 \mathrm{~ms}, \mathrm{p}=0.13$, Fig. 3A). In expect late blocks, the directionality of the trend remained the same, but again, did not reach significance ( 873 vs. $844 \mathrm{~ms}, \mathrm{p}=0.16$ for the primary; 883 vs. $890 \mathrm{~ms}, \mathrm{p}=0.72$, for secondary modality targets, Fig. 3B). To test the influence of the spatial location of the stimuli upon the pattern of results, the factor of spatial alignment was introduced. Yet, neither the main effect of spatial alignment itself, nor any of its interactions reached significance, suggesting that the relative spatial positioning of the stimuli was not relevant in this particular context.

The results provided more evidence in favour of the decoupling hypothesis than in experiment 1 , but the overall pattern was not statistically clear-cut. We decided to run an exploratory analysis under the post-hoc hypothesis that our observed effect might be stronger in the first half of the experiment (first 3 blocks) than in the second. The reasoning is that, as some studies point out, participants might have to unlearn the set of temporal probabilities from the initial part of the experiment, and relearn the new temporal properties of the second half of the experiment with the ensuing costs (e.g. Thomaschke et. al, 2015). If this is true, weakened patterns of temporal orienting in the second half of the experiment seems to be a logical conclusion. As a first step, we ran an ANOVA with the additional between-participant factor of Starting Block (expect early or late). We found that the Starting Block significantly interacted with the factors of block type $\left(\mathrm{F}_{1,24}=30.72 ; \mathrm{p}<0.01\right)$ and with the factors of block type and modality prevalence $(F 1,24=1.48, p<0.01)$, showing that responses get 
naturally faster over the course of the experiment. Importantly, the resulting reaction time decrease from first to second blocks led to a generalized equalization of reaction times between primary and secondary targets (early group: primary from $929 \mathrm{~ms}$ to $853 \mathrm{~ms}$ and secondary from $1004 \mathrm{~ms}$ to $843 \mathrm{~ms}$; late group: from primary $863 \mathrm{~ms}$ to $819 \mathrm{~ms}$ and secondary from $930 \mathrm{~ms}$ to $816 \mathrm{~ms}$ ). In other words, a possible improvement leading to a ceiling effect in terms of reaction speed might have dampened the behavioural effects toward the second half of the experiment. Since we could neither exclude the possibility that participants had not fully unlearned the temporal pattern of the first experimental half, or that the behavioural improvement had led to a ceiling effect, we decided to run an ANOVA only including the first three experimental blocks over all participants with the factors of: time expectation (now a between subject factor), spatial alignment, modality prevalence, and onset time.

Despite halving the number of trials, the new ANOVA on first-blocks data revealed a highly significant interaction between time expectation, modality prevalence, and onset time $\left(\mathrm{F}_{1,24}=9.91, \mathrm{p}<0.01\right)$, the predicted pattern in our original hypothesis. Post-hoc analyses revealed that participants of the expect early group responded significantly faster to early primary targets than to late primary targets ( $894 \mathrm{~ms}$ vs. $964 \mathrm{~ms}, \mathrm{p}=0.043$ ); whereas, for secondary modality targets, an opposite trend emerged (faster responses towards late than early targets; 1033 ms vs. $975 \mathrm{~ms}, \mathrm{p}=0.089$ ). In contrast, no such pattern was observed for participants of the expect late group. Additionally, we found a main effect of modality prevalence $\left(\mathrm{F}_{1,24}=6.84, \mathrm{p}=0.015\right)$, showing faster responses to primary (896ms) compared to secondary modality targets $(967 \mathrm{~ms})$. This effect was additionally modulated by spatial alignment $\left(\mathrm{F}_{1,24}=5.06, \mathrm{p}=0.034\right)$, since only the unaligned group showed this difference ( $\mathrm{p}<0.01$ for the unaligned group vs. $\mathrm{p}=0.79$ for the aligned group). However, since, no other main effect or interaction could be observed, the data again shows no effect of spatial alignment on the expression of temporal attention.

Finally, to check whether the auditory stimuli of experiment 2 were indeed more difficult than in experiment 1 , we compared the data of the spatial misaligned group of experiment 2 (i.e., the most comparable dataset), to the audition group of experiment 1 (both, $\mathrm{N}=14$ ) by running an ANOVA with the between-group factor of task difficulty and the within-group factors of modality prevalence, onset time, and block type. This analysis revealed an interaction between task difficulty and modality prevalence $\left(\mathrm{F}_{1,24}=5.41, \mathrm{p}=0.028\right)$. While there was a large significant reaction time difference for the primary (audition) and secondary (touch) modality 
in experiment 1 (easy, 807 vs. 992 ms, p<0.01), the difference was much smaller for the data of experiment 2, reaching only a marginally significant trend (difficult, 893 vs. 962 ms, p=0.059). Hence, overall RTs were far less imbalanced in experiment 2 than in experiment 1.

\section{Accuracy}

Accuracy was high on average ( $90.2 \%$ correct responses). We also observed a significant main effect of modality prevalence $\left(\mathrm{F}_{1,24}=13.55, \mathrm{p}<0.01\right)$, in which responses towards primary modality targets were more accurate $(93.9 \%)$ than secondary modality targets $(86.4 \%)$. Additionally, we found an interaction between the block type and onset time $(\mathrm{F} 1,14=6.32, \mathrm{p}=0.019)$. Post-hoc analyses revealed that for expect late onset trials, participants responded slightly less accurately $(\mathrm{p}=0.038)$ at the early time point $(88.9 \%)$ than at the late time point $(91.6 \%)$. Similar to what occurred in the reaction time data, no effect of spatial alignment was found.

An ANOVA comparing the data of experiment 1 and 2 with the between-group factor of task difficulty and the within-group factors of modality prevalence, onset time, and block type revealed no effect of the task difficulty on accuracy.

\section{Discussion}

In experiment 2 , the difficulty of auditory targets was rendered similar to that of tactile targets by decreasing the loudness of the auditory stimulus. Responses towards the primary auditory targets were also slower and (marginally) less accurate than in experiment 1. Critically, we found a significant three-way interaction between modality prevalence, onset time, and block type. While the primary auditory modality showed a strong trend towards faster responses at the expected time point, the secondary tactile stimuli followed an opposite trend. In a post-hoc exploratory analysis, we found that these trends were fully significant when considering data from the first half of the experiment alone. This provides some evidence in favour of cross-modal temporal decoupling, and that the pattern might not have been observed in experiment 1 because of the imbalance in task difficulty between the modalities. While there was no significant effect of the auditory difficulty on accuracy, the reaction times towards auditory targets in experiment 2 were significantly higher compared to experiment 1, suggesting an increased difficulty. Therefore, these results suggest that the strong modality prevalence effect 
in experiment 1 could have potentially masked any effects of the interaction between attention-to-time and attention modality due to its dominance.

Additionally, in experiment 2, we also investigated whether the lack of cross-modal coupling in experiment 1 could be explained by the spatial misalignment of the auditory and tactile stimuli. As we expected, however, spatial (mis)alignment had no sizable impact on the pattern of results (see the discussion of experiment 1), suggesting that the now observed cross-modal temporal decoupling is not obliterated by a slight misalignment between the tactile and auditory target locations (within the values used here).

\section{General Discussion}

The main finding of the present study is that orienting attention in time allows cross-modal temporal decoupling (Mühlberg et al., 2014). Cross-modal decoupling means, as it was the case in the present study, that temporal orienting in each modality can unfold according to different temporal expectation patterns. This is in stark contrast to the hypothesis of cross-modal temporal coupling, whereby temporal orienting in all modalities would be dominated by the expectation pattern of one (most relevant) modality, as it was previously suggested in an earlier study (Lange \& Röder, 2006). Because contradictory results from two previous studies regarding crossmodal temporal attention had been obtained using different modality combinations, an open question was whether cross-modal coupling and decoupling may manifest selectively for audio-tactile pairings, but not for visuo-tactile pairs. Hence, here we used a modified version of the experiment of Mühlberg et al. (2014) with auditory and tactile stimuli and observed that, in spite of the involvement of the auditory modality, which is the most reliable modality in terms of time perception (e.g., Ball et al., 2018; Bresciani et al., 2005; Fendrich \& Corballis, 2001; Morein-Zamir et al., 2003), behaviour was mostly consistent with cross-modal temporal decoupling. The fact that decoupling is found between at least two pairs of modalities suggests therefore, that it might be a general property of temporal attention.

The result of cross-modal decoupling in time stands in contrast to cross-modal spatial attention in multisensory contexts, where coupling between modalities seems to be the norm (Driver \& Spence, 1994, 1998b; Kennett et al., 2001; Spence \& Driver, 1996). In cross-modal spatial attention, it is the most frequent case that different 
sensory modalities follow a common orienting pattern, consistent with one dominant modality (see Driver \& Spence, 1994; Spence \& Driver, 1996; Spence et al., 2000; Teder-Sälejärvi et al., 1999, regard however Lloyd et al., 2003 and Soto-Faraco et al., 2005 for possible exceptions), suggesting a dominance of spatial attention over modality attention. This finding is supported by the investigation of pre-stimulus oscillations in brain activity using MEG, which have shown a similar lateralization pattern of alpha and beta oscillations in the occipital and central cortex, regardless of whether visual or tactile attention was oriented in space (Bauer et al., 2012). On the other hand, the interplay between temporal and modality attention seems to be more flexible than the interplay between spatial and modality attention (Keil et al., 2016). An EEG study from Keil et al. (2016) found that attention towards a targets modality leads to a different and independent pattern of pre-stimulus activity in different frequency ranges and brain areas, then focussing attention towards the temporal properties of the target. An interaction between attention to time and modality, was expressed by a modulation of prestimulus theta band activity (see also, Pomper et al., 2015, using the same data set). Both, Keil et al. and Pomper et al. found that modality attention leads to modulations in the alpha and beta band within sensory cortices, and temporal attention leads to activation of the motor cortex in the beta and delta bands. While the studies of Bauer et al. (2012), Keil et al. (2016) and Pomper et al. (2015) are not directly comparable in task design, their basic finding aligns well with the present results, and supports the hypothesis that there are intrinsic differences in the way cross-modal attention occurs during spatial and temporal orienting. A recent study of the same group (Keil et al., 2017) used simultaneously presented, visual and tactile stimuli after a regular or irregular temporal interval and participants were instructed to orient either to the visual or the tactile stimulus via an auditory cue. Analysing the ERPs, the authors found that the effects of temporal orienting occurred already around $25-75 \mathrm{~ms}$ after the stimulus onset; whereas, the effects of modality attention were observed around 100ms later.

Several other differences between the neural correlates of attention to time and attention to space exist. Spatial attention is associated with a lateralization in the alpha frequency range (for vision or audition) or alpha/beta frequency range (for touch), in particular through inter-hemispheric imbalance in amplitude (see e.g. Jensen \& Mazaheri, 2010; Ruzzoli \& Soto-Faraco, 2014). Temporal attention leads to a lateralization of the beta band activity, which holds true for different modalities such as touch (van Ede et al., 2011) and audition (Todorovic et al., 2015). Spatial and temporal attention, despite sharing some similar patterns of cortical activity in the fMRI, also leads to non-overlapping activations. For example, spatial attention, but not temporal attention, 
usually activates areas such as the frontal eye field (Liu et al., 2014; Yang \& Mayer, 2013); whereas, activations such as in the superior occipital gyrus or the cerebellum seem to be rather related to temporal attention (Davranche et al., 2011; Li et al., 2012). All these studies suggest that there might be important differences in the mechanisms underlying temporal and spatial attention, and our results would support such a hypothesis.

As mentioned in the introduction, in order to induce (e.g., motivate) attention orienting, the present study used a foreperiod paradigm to manipulate the temporal likelihoods, which does lead to modulations in attention and expectation. Both, attention and expectation, can lead to the undistinguishable behavioural expression, but fairly different neural expressions (e.g. Lange, 2013). When properly dissociated, (temporal) attention usually leads to increased neural activity in response to the attended target (Schroeder \& Lakatos, 2009); whereas, expectation often leads to decreased or rather sharpened neural activity in response to the expected target (Bendixen et al., 2012; Schwartze et al., 2013). ${ }^{3}$ Evidence suggests that, when a study incorporates both, attention and expectation, then attention tends to reverse the effects of expectation (Kok et al., 2012; Todorovic et al., 2015), thus, one could conclude that one observes predominantly the effect of attention in such a design. However, a direct distinction would not be possible in a purely behavioural design. Our study employs time-based expectation as a means to induce attention shifts (following the paradigm used by previous studies addressing cross-modal coupling and decoupling (Lange \& Röder, 2006; Mühlberg et al., 2014). Thus, time-based expectancy, as well as explicit instruction, was used as a way to manipulate the participants' expectation about an upcoming target. Another question is in how far our manipulation can be classified as a time-based attention or as temporal attention study. Time-based orienting of attention is defined as the anticipation of a certain event feature in time, whereas temporal orienting represents an orientation towards time itself (Thomaschke \& Dreisbach, 2015). Time-based expectations are solely dependent on the temporal likelihoods of an event, not on the additional deployment of attention upon it (Thomaschke et al., 2011), and can be mainly explained by motor preparations (Volberg and Thomaschke, 2017). If one would classify our study in the light of time-based expectancy, then our results would, however, be in contrast with the results of Volberg and Thomaschke (2017), since they suggest that time-based expectancies express

\footnotetext{
${ }^{3}$ Responses towards expected stimuli are eliciting a weaker N100 component than unexpected stimuli (Lange, 2009) and reduced fMRI activity (Kok et al., 2012). This reduced activity is thereby caused through a suppression of the activity of neurons encoding for unexpected events, leading to a sharpening of neural activity (Kok, Jehee, et al., 2012).
} 
differently for modalities with different temporal probabilities. However, within our study participants had to focus their attention upon time, while also employing time-based expectations for the individual modalities. In other words, we assume that participants did not only anticipate different events at different time points, but they also oriented their attention towards the probable time point, given the overall target likelihoods as well as the explicit instructions received. Thus, the focus of our study can be seen as the interplay between orienting attention in time and towards modality via time-based expectancies, and therefore are not in contrast with Volberg and Thomaschke (2017).

To conclude, our results support the hypothesis that cross-modal temporal decoupling is a possible general property of temporal attention. Hence, it is not dependent on the concrete modalities. Although the effects seen here are small and sometimes marginal, which should call for cautious interpretation of the present results, evidence for cross-modal coupling was non-existent. Altogether, this pattern could suggest that crossmodal decoupling-in-time might be a differential feature of the expression of attention orienting in time, compared to the cross-modal coupled orienting typically seen in spatial attention. Various neuroimaging studies, together with the present behavioural data, seem to align well with this idea.

\section{Acknowledgements}

This research was funded by the Spanish Ministry of Science and Innovation (PSI2016-75558-P(AEI/FEDER)), the Comissionat per a Universitats i Recerca del DIUE-Generalitat de Catalunya (2014SGR856) and the European Research Council (StG-2010 263145) to S.S.F.

\section{Compliance with Ethical Standards}

\section{Conflict of Interest}

The authors declare that they have no conflict of interest.

\section{Research involving human participants}

All research conducted in this paper was conducted in accordance to the 1964 Declaration of Helsinki and approved by the ethics committee CEIC Parc de Salut Mar (University Pompeu Fabra, Barcelona, Spain). 
Informed consent

All participants gave written an informed consent to participate in the study.

\section{References}

Aufschnaiter, S., Kiesel, A., \& Thomaschke, R. (2017). Transfer of time-based task expectancy across different timing environments. Psychological Research. https://doi.org/10.1007/s00426-017-0895-1

Ball, F., Michels, L. E., Thiele, C., \& Noesselt, T. (2018). The role of multisensory interplay in enabling temporal expectations. Cognition, 170(January 2018), 130-146. https://doi.org/10.1016/j.cognition.2017.09.015

Bauer, M., Kennett, S., \& Driver, J. (2012). Attentional selection of location and modality in vision and touch modulates low-frequency activity in associated sensory cortices. Journal of Neurophysiology, 107(9), 2342-2351. https://doi.org/10.1152/jn.00973.2011

Bendixen, A., SanMiguel, I., \& Schröger, E. (2012). Early electrophysiological indicators for predictive processing in audition: A review. International Journal of Psychophysiology, 83(2), 120-131. https://doi.org/10.1016/j.ijpsycho.2011.08.003

Correa, A., Lupiáñez, J., Milliken, B., \& Tudela, P. (2004). Endogenous temporal orienting of attention in detection and discrimination tasks. Perception \& Psychophysics, 66(2), 264-278. https://doi.org/10.3758/BF03194878

Correa, A., Lupiáñez, J., \& Tudela, P. (2005). Attentional preparation based on temporal expectancy modulates processing at the perceptual level. Psychonomic Bulletin \& Review, 12(2), 328-334. https://doi.org/10.3758/BF03196380

Correa, A., \& Nobre, A. C. (2008). Spatial and temporal acuity of visual perception can be enhanced selectively by attentional set. Experimental Brain Research, 189(3), 339-344. https://doi.org/10.1007/s00221-008-1429-2

Coull, J. T., \& Nobre, A. C. (1998). Where and when to pay attention: the neural systems for directing attention to spatial locations and to time intervals as revealed by both PET and fMRI. The Journal of Neuroscience, 18(18), 7426-7435. Retrieved from http://www.ncbi.nlm.nih.gov/pubmed/9736662

Cravo, A. M., Rohenkohl, G., Wyart, V., \& Nobre, A. C. (2013). Temporal expectation enhances contrast sensitivity by phase entrainment of low-frequency oscillations in visual cortex. The Journal of Neuroscience, 33(9), 4002-4010. https://doi.org/10.1523/JNEUROSCI.4675-12.2013

Davranche, K., Nazarian, B., Vidal, F., \& Coull, J. T. (2011). Orienting attention in time activates left intraparietal sulcus for both perceptual and motor task goals. Journal of Cognitive Neuroscience, 23(11), 3318-3330. https://doi.org/10.1162/jocn_a_00030

Desimone, R., \& Duncan, J. (1995). Neural Mechanisms of Selective Visual Attention. Annual Reviews of Neuroscience, 18, 193-222. Retrieved from http://www.annualreviews.org/doi/pdf/10.1146/annurev.ne.18.030195.001205

Driver, J., \& Spence, C. (1994). Spatial synergies between auditory and visual attention. In C. Umiltà \& M. Moscovitch (Eds.), Attention and performance XV: Conscious and nonconscious information processing (pp. 311-331). Cambridge, MA: MIT Press.

Driver, J., \& Spence, C. (1998a). Attention and the crossmodal construction of space. Trends in Cognitive Sciences, 2(7), 254-262. https://doi.org/10.1016/S1364-6613(98)01188-7

Driver, J., \& Spence, C. (1998b). Cross-modal links in spatial attention. Philosophical Transactions of the Royal Society of London. Series B, Biological Sciences, 353(1373), 1319-1331. https://doi.org/10.1098/rstb.1998.0286 
Eimer, M. (1999). Can attention be directed to opposite locations in different modalities? An ERP study. Clinical Neurophysiology, 110(7), 1252-1259. Retrieved from http://www.ncbi.nlm.nih.gov/pubmed/10423190

Eimer, M., \& Driver, J. (2000). An event-related brain potential study of cross-modal links in spatial attention between vision and touch. Psychophysiology, 37(5), 697-705. Retrieved from http://www.ncbi.nlm.nih.gov/pubmed/11037046

Eimer, M., van Velzen, J., \& Driver, J. (2002). Cross-modal interactions between audition, touch, and vision in endogenous spatial attention: ERP evidence on preparatory states and sensory modulations. Journal of Cognitive Neuroscience, 14(2), 254-271. https://doi.org/10.1162/089892902317236885

Griffin, I. C., Miniussi, C., \& Nobre, A. C. (2001). Orienting Attention in Time. Frontiers in Bioscience, 6, $660-671$.

Jensen, O., \& Mazaheri, A. (2010). Shaping functional architecture by oscillatory alpha activity: gating by inhibition. Frontiers in Human Neuroscience, 4(November), 186. https://doi.org/10.3389/fnhum.2010.00186

Keil, J., Pomper, U., \& Feuerbach, N. (2017). Temporal orienting precedes intersensory attention and has opposing effects on early evoked brain activity. NeuroImage, 148, 230-239. https://doi.org/10.1016/j.neuroimage.2017.01.039

Keil, J., Pomper, U., \& Senkowski, D. (2016). Distinct patterns of local oscillatory activity and functional connectivity underlie intersensory attention and temporal prediction. Cortex, 74(November), 277-288. https://doi.org/10.1016/j.cortex.2015.10.023

Kennett, S., Eimer, M., Spence, C., \& Driver, J. (2001). Tactile-visual links in exogenous spatial attention under different postures: convergent evidence from psychophysics and ERPs. Journal of Cognitive Neuroscience, 13(4), 462-478. Retrieved from http://www.ncbi.nlm.nih.gov/pubmed/11388920

Klein, R. M. (1977). Attention and Visual Dominance: A Chronometric Analysis. Journal of Experimental Psychology: Human Perception and Performance, 3(3), 365-378.

Kok, P., Jehee, J. F. M., \& de Lange, F. P. (2012). Less is more: expectation sharpens representations in the primary visual cortex. Neuron, 75(2), 265-270. https://doi.org/10.1016/j.neuron.2012.04.034

Kok, P., Rahnev, D., Jehee, J. F. M., Lau, H. C., \& de Lange, F. P. (2012). Attention reverses the effect of prediction in silencing sensory signals. Cerebral Cortex, 22(9), 2197-2206. https://doi.org/10.1093/cercor/bhr310

Kubovy, M. (1988). Should we resist the seductiveness of the space is to time as vision is to audition analogy? Journal of Experimental Psychology: Human Perception and Performance, 14(2), 318-320.

Lampar, A., \& Lange, K. (2011). Effects of temporal trial-by-trial cuing on early and late stages of auditory processing: Evidence from event-related potentials. Attention, Perception \& Psychophysics, 73, 1916 1933. https://doi.org/10.3758/s13414-011-0149-z

Lange, K. (2009). Brain correlates of early auditory processing are attenuated by expectations for time and pitch. Brain and Cognition, 69(1), 127-137. https://doi.org/10.1016/j.bandc.2008.06.004

Lange, K. (2012). The N1 effect of temporal attention is independent of sound location and intensity: implications for possible mechanisms of temporal attention. Psychophysiology, 49(11), 1468-1480. https://doi.org/10.1111/j.1469-8986.2012.01460.x

Lange, K. (2013). The ups and downs of temporal orienting: a review of auditory temporal orienting studies and a model associating the heterogeneous findings on the auditory N1 with opposite effects of attention and prediction. Frontiers in Human Neuroscience, 7(June), 263. https://doi.org/10.3389/fnhum.2013.00263

Lange, K., Krämer, U. M., \& Röder, B. (2006). Attending points in time and space. Experimental Brain Research, 173(1), 130-140. https://doi.org/10.1007/s00221-006-0372-3 
Lange, K., \& Röder, B. (2006). Orienting attention to points in time improves stimulus processing both within and across modalities. Journal of Cognitive Neuroscience, 18(5), 715-729. https://doi.org/10.1162/jocn.2006.18.5.715

Lange, K., Rösler, F., \& Röder, B. (2003). Early processing stages are modulated when auditory stimuli are presented at an attended moment in time: an event-related potential study. Psychophysiology, 40(5), 806-817. Retrieved from http://www.ncbi.nlm.nih.gov/pubmed/14696734

Li, C., Chen, K., Han, H., Chui, D., \& Wu, J. (2012). An FMRI study of the neural systems involved in visually cued auditory top-down spatial and temporal attention. PloS One, 7(11), e49948. https://doi.org/10.1371/journal.pone.0049948

Liu, Y., Bengson, J., Huang, H., Mangun, G. R., \& Ding, M. (2014). Top-down Modulation of Neural Activity in Anticipatory Visual Attention: Control Mechanisms Revealed by Simultaneous EEG-fMRI. Cerebral Cortex (New York, N.Y. : 1991), (February), 517-529. https://doi.org/10.1093/cercor/bhu204

Lloyd, D. M., Merat, N., McGlone, F., \& Spence, C. (2003). Crossmodal links between audition and touch in covert endogenous spatial attention. Perception \& Psychophysics, 65(6), 901-924.

Los, S. A., Knol, D. L., \& Boers, R. M. (2001). The foreperiod effect revisited: Conditioning as a basis for nonspecific preparation. Acta Psychologica, 106(1-2), 121-145. https://doi.org/10.1016/S00016918(00)00029-9

Macaluso, E. (2010). Orienting of spatial attention and the interplay between the senses. Cortex, 46(3), 282297. https://doi.org/10.1016/j.cortex.2009.05.010

Macaluso, E., \& Driver, J. (2005). Multisensory spatial interactions: a window onto functional integration in the human brain. Trends in Neurosciences, 28(5), 264-271. https://doi.org/10.1016/j.tins.2005.03.008

Macaluso, E., Frith, C. D., \& Driver, J. (2000). Modulation of Human Visual Cortex by Crossmodal Spatial Attention. Science, 289(5482), 1206-1208. https://doi.org/10.1126/science.289.5482.1206

Miniussi, C., Wilding, E. L., Coull, J. T., \& Nobre, A. C. (1999). Orienting attention in time. Modulation of brain potentials. Brain, 122, 1507-1518. Retrieved from http://www.ncbi.nlm.nih.gov/pubmed/10430834

Mühlberg, S., Oriolo, G., \& Soto-Faraco, S. (2014). Cross-modal decoupling in temporal attention. European Journal of Neuroscience, 39, 2089-2097. https://doi.org/10.1111/ejn.12563

Nobre, A. C., \& Rohenkohl, G. (2014). Time for the Fourth Dimension of Attention. In A. C. Nobre \& S. Kastner (Eds.), The Oxford Handbook of Attention (pp. 676-724). Oxford University Press. https://doi.org/10.1093/oxfordhb/9780199675111.013.036

Pomper, U., Keil, J., Foxe, J. J., \& Senkowski, D. (2015). Intersensory selective attention and temporal orienting operate in parallel and are instantiated in spatially distinct sensory and motor cortices. Human Brain Mapping, 3259(April), 3246-3259. https://doi.org/10.1002/hbm.22845

Posner, M. I., Nissen, M. J., \& Klein, R. M. (1976). Visual dominance: An information-processing account of its origins and significance. Psychological Review, 83(2), 157-171.

Rohenkohl, G., Cravo, A. M., Wyart, V., \& Nobre, A. C. (2012). Temporal expectation improves the quality of sensory information. The Journal of Neuroscience, 32(24), 8424-8428. https://doi.org/10.1523/JNEUROSCI.0804-12.2012

Ruzzoli, M., \& Soto-Faraco, S. (2014). Alpha stimulation of the human parietal cortex attunes tactile perception to external space. Current Biology, 24(3), 329-332. https://doi.org/10.1016/j.cub.2013.12.029

Sambo, C. F., \& Forster, B. (2011). When far is near: ERP correlates of crossmodal spatial interactions between tactile and mirror-reflected visual stimuli. Neuroscience Letters, 500(1), 10-15. https://doi.org/10.1016/j.neulet.2011.05.233

Sanders, L. D., \& Astheimer, L. B. (2008). Temporally selective attention modulates early perceptual 
processing: Event-related potential evidence. Perception \& Psychophysics, 70(4), 732-742. https://doi.org/10.3758/PP.70.4.732

Santangelo, V., Fagioli, S., \& Macaluso, E. (2010). The costs of monitoring simultaneously two sensory modalities decrease when dividing attention in space. NeuroImage, 49(3), 2717-2727. https://doi.org/10.1016/j.neuroimage.2009.10.061

Schroeder, C. E., \& Lakatos, P. (2009). Low-frequency neuronal oscillations as instruments of sensory selection. Trends in Neurosciences, 32(1), 9-18. https://doi.org/10.1016/j.tins.2008.09.012

Schröter, H., Birngruber, T., Bratzke, D., Miller, J., \& Ulrich, R. (2015). Task predictability influences the variable foreperiod effect: evidence of task-specific temporal preparation. Psychological Research, 79(2), 230-237. https://doi.org/10.1007/s00426-014-0550-z

Schwartze, M., Farrugia, N., \& Kotz, S. A. (2013). Dissociation of formal and temporal predictability in early auditory evoked potentials. Neuropsychologia, 51(2), 320-325. https://doi.org/10.1016/j.neuropsychologia.2012.09.037

Soto-Faraco, S., Morein-Zamir, S., \& Kingstone, A. (2005). On audiovisual spatial synergy: the fragility of the phenomenon. Perception \& Psychophysicssychophysics, 67(3), 444-457. Retrieved from http://www.ncbi.nlm.nih.gov/pubmed/16119393

Spence, C., \& Driver, J. (1996). Audiovisual Links in Endogenous Covert Spatial Attention. Journal of Experimental Psychology: Human Perception and Performance, 22(4), 1005-1031.

Spence, C., \& Driver, J. (1997). On measuring selective attention to an expected sensory modality. Perception \& Psychophysics, 59(3), 389-403. Retrieved from http://www.ncbi.nlm.nih.gov/pubmed/9136269

Spence, C., Nicholls, M. E. R., \& Driver, J. (2001). The cost of expecting events in the wrong sensory modality. Perception \& Psychophysics, 63(2), 330-336. Retrieved from http://www.ncbi.nlm.nih.gov/pubmed/11281107

Spence, C., Pavani, F., \& Driver, J. (2000). Crossmodal Links between Vision and Touch in Covert Endogenous Spatial Attention. Journal of Experimental Psychology: Human Perception and Performance, 26(4), 1298-1319.

Spitzer, H., Desimone, R., \& Moran, J. (1988). Increased Attention Enhances Both Behavioral and Neuronal Performance. Science, 240(4850), 338-340.

Störmer, V. S., McDonald, J. J., \& Hillyard, S. a. (2009). Cross-modal cueing of attention alters appearance and early cortical processing of visual stimuli. Proceedings of the National Academy of Sciences of the United States of America, 106(52), 22456-22461. https://doi.org/10.1073/pnas.0907573106

Summerfield, C., \& Egner, T. (2009). Expectation (and attention) in visual cognition. Trends in Cognitive Sciences, 13(9), 403-409. https://doi.org/10.1016/j.tics.2009.06.003

Tang, X., Wu, J., \& Shen, Y. (2016). The interactions of multisensory integration with endogenous and exogenous attention. Neuroscience \& Biobehavioral Reviews, 61, 208-224. https://doi.org/10.1016/j.neubiorev.2015.11.002

Teder-Sälejärvi, W. A., Münte, T. F., Sperlich, F., \& Hillyard, S. A. (1999). Intra-modal and cross-modal spatial attention to auditory and visual stimuli. An event-related brain potential study. Brain Research. Cognitive Brain Research, 8(3), 327-343. https://doi.org/S0926641099000373 [pii]

ten Oever, S., Romei, V., van Atteveldt, N., Soto-Faraco, S., Murray, M. M., \& Matusz, P. J. (2016). The COGs (context, object, and goals) in multisensory processing. Experimental Brain Research, 234(5), 1307-1323. https://doi.org/10.1007/s00221-016-4590-z

Thomaschke, R., \& Dreisbach, G. (2015). The Time-Event Correlation Effect Is Due to Temporal Expectancy, Not to Partial Transition Costs. Journal of Experimental Psychology: Human Perception and Performance, 4l(1), 196-218. https://doi.org/10.1037/a0038328

Thomaschke, R., Kunchulia, M., \& Dreisbach, G. (2015). Time-based event expectations employ relative, not 
absolute, representations of time. Psychonomic Bulletin \& Review, 22, 890-895.

https://doi.org/10.3758/s13423-014-0710-6

Thomaschke, R., Wagener, A., Kiesel, A., \& Hoffmann, J. (2011). The scope and precision of specific temporal expectancy: evidence from a variable foreperiod paradigm. Attention, Perception \& Psychophysics, 73, 953-964. https://doi.org/10.3758/s13414-010-0079-1

Todorovic, A., Schoffelen, J. M., Van Ede, F., Maris, E., \& De Lange, F. P. (2015). Temporal expectation and attention jointly modulate auditory oscillatory activity in the beta band. PLOS ONE, 10(3), 1-16. https://doi.org/10.1371/journal.pone.0120288

Trenner, M. U., Heekeren, H. R., Bauer, M., Rössner, K., Wenzel, R., Villringer, A., \& Fahle, M. (2008). What happens in between? Human oscillatory brain activity related to crossmodal spatial cueing. PloS One, 3(1), e1467. https://doi.org/10.1371/journal.pone.0001467

Treue, S. (2003). Visual attention: the where, what, how and why of saliency. Current Opinion in Neurobiology, 13(4), 428-432. https://doi.org/10.1016/S0959-4388(03)00105-3

van Ede, F., de Lange, F., Jensen, O., \& Maris, E. (2011). Orienting Attention to an Upcoming Tactile Event Involves a Spatially and Temporally Specific Modulation of Sensorimotor Alpha- and Beta-Band Oscillations. Journal of Neuroscience, 31(6), 2016-2024. https://doi.org/10.1523/JNEUROSCI.563010.2011

VanRullen, R., Zoefel, B., \& Ilhan, B. (2014). On the cyclic nature of perception in vision versus audition. Philosophical Transactions of the Royal Society of London. Series B, Biological Sciences, 369, 20130214. https://doi.org/10.1098/rstb.2013.0214

Volberg, G., \& Thomaschke, R. (2017). Time-based expectations entail preparatory motor activity. Cortex, 92, 261-270. https://doi.org/10.1016/j.cortex.2017.04.019

Wagener, A., \& Hoffmann, J. (2010). Temporal cueing of target-identity and target-location. Experimental Psychology, 57(6), 436-445. https://doi.org/10.1027/1618-3169/a000054

Welch, R. B., \& Warren, D. H. (1980). Immediate perceptual response to intersensory discrepancy. Psychological Bulletin, 88(3), 638-687. https://doi.org/http://dx.doi.org/10.1037/0033-2909.88.3.638

Yang, Z., \& Mayer, A. R. (2014). An event-related FMRI study of exogenous orienting across vision and audition. Human Brain Mapping, 35(3), 964-974. https://doi.org/10.1002/hbm.22227 
Fig. 1: Schematic description of the task. Every trial started with a colour change of a visual arrow cue situated left or right from the fixation cross. After $1.2 \mathrm{~s}$ or $2.4 \mathrm{~s}$, an auditory or a tactile target could appear. The likelihood of appearance of each combination of time-point and modality differed in order to drive attention to time and modality in a certain direction. One of the two modalities was far more likely to appear (constant between participants) and the target was more likely to appear at one of the time points (constant between blocks). Importantly, while targets of the more likely, primary modality would be mostly presented at the more likely, expected time point, these targets were presented as a minority at the unexpected time point, where most targets were of the secondary modality. In order to prevent complete temporal certainty, some trials contained no target at all.

Fig. 2: Overview over the mean reaction time trends in experiment 1. Dashed lines represent the observed trends $(\mathrm{p}<0.1)$, while significant differences are marked through a solid line and an asterisk $(\mathrm{p}<0.05)$. Numbers next to each data point represent its respective accuracy. (A) Interaction between modality prevalence, onset time, and block type. A non-significant trend towards faster responses at the early (expected) time point in the expect early blocks for the primary modality was observed ( $812 \mathrm{~ms}$ vs. $866 \mathrm{~ms}, \mathrm{p}=0.065)$. Since the interaction seems to be further modulated by the primary modality $(\mathrm{p}=0.061)$, the pattern was further divided for the different modality groups. (B) Interaction between modality prevalence, onset time, and block type for the touch group. Participants responded significantly ( $836 \mathrm{~ms}$ vs. $919 \mathrm{~ms}, \mathrm{p}=0.046$, expect early blocks) or nearly significantly (916 ms vs. $841 \mathrm{~ms}, \mathrm{p}=0.068$, expect late blocks) towards the primary modality at the expected time points, while no such pattern was observed for the secondary modality (898 ms vs. $894 \mathrm{~ms}$ in the expect early blocks and $884 \mathrm{~ms}$ vs. $941 \mathrm{~ms}$ in the expect late blocks). (C) Interaction between modality prevalence, onset time, and block type for the audition group. No significant effects or trends could be observed.

Fig. 3: Interaction between modality prevalence, block type and onset time in experiment 2. Dashed lines represent the observed trends $(\mathrm{p}<0.1)$. Numbers next to each data point represent its respective accuracy. (A) Interaction between modality prevalence and onset time in the expect early blocks. Participants tended to 
respond faster towards the primary modality at the early (expected) time point $(\mathrm{p}=0.069)$, while responses towards the other, secondary modality had the opposite directionality $(\mathrm{p}=0.13)$. (B) Interaction between modality prevalence and onset time in the expect late blocks. Participants were slightly, albeit insignificantly faster for the primary modality at the late (expected) time point $(\mathrm{p}=0.16)$. No difference for reaction time at the two onset times was observed for the secondary modality $(\mathrm{p}=0.72)$. 


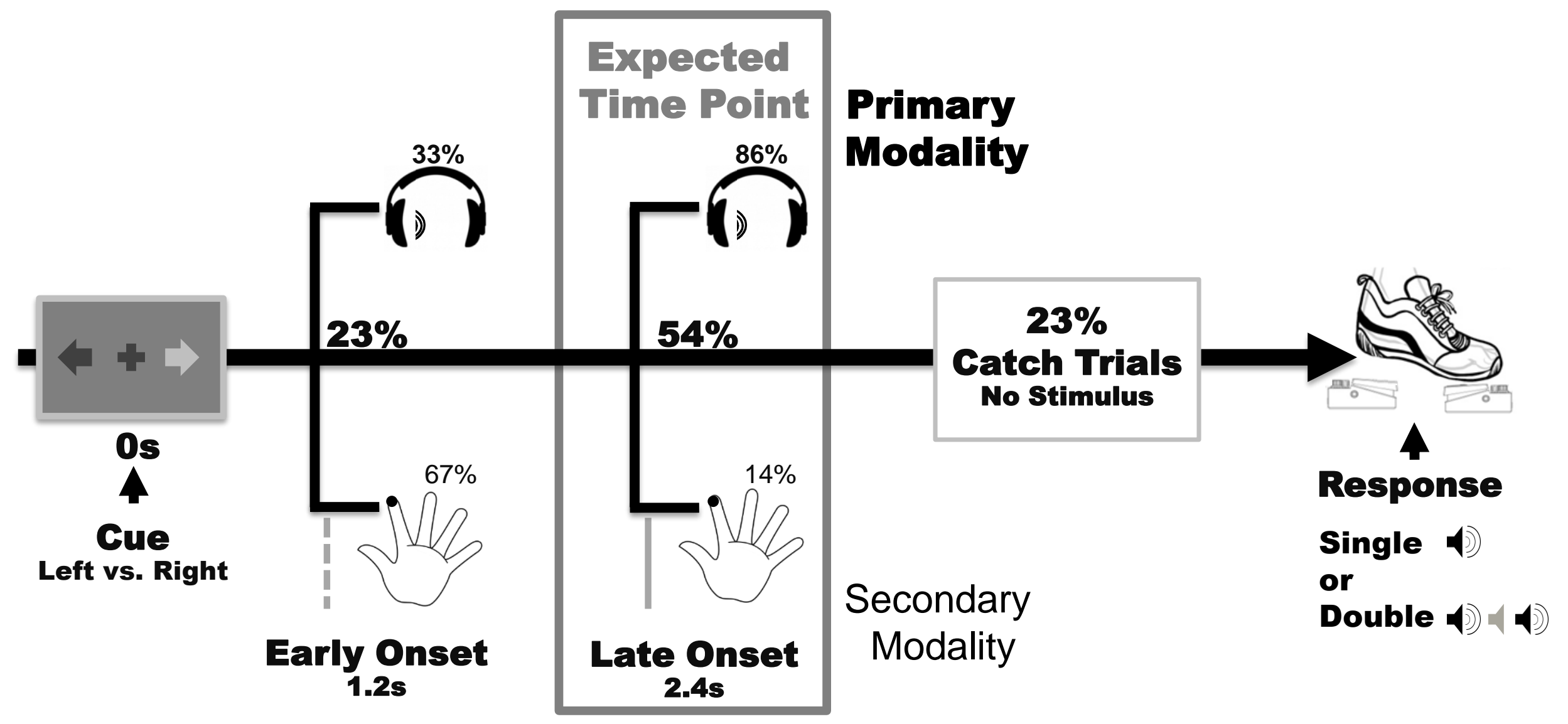




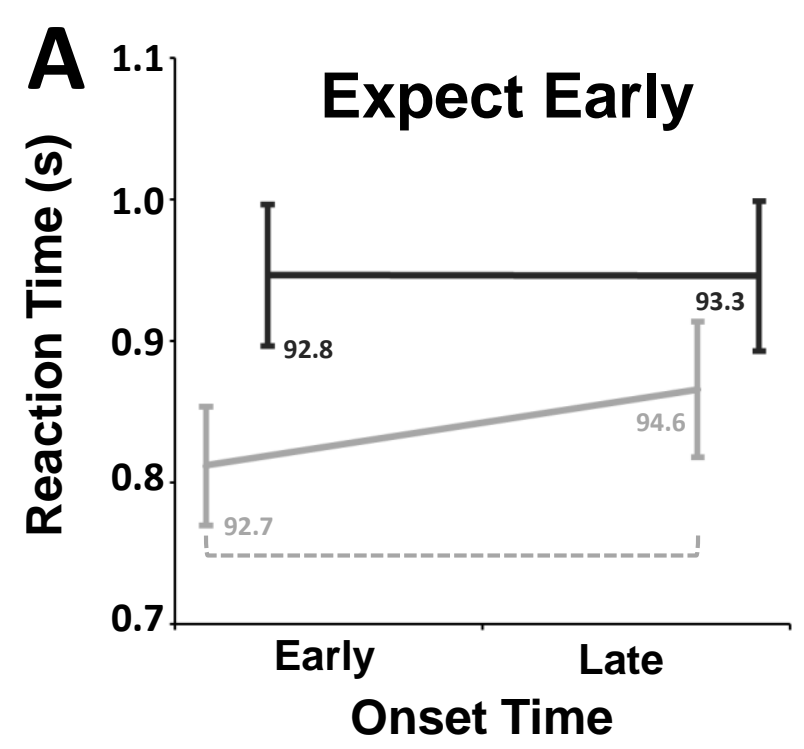

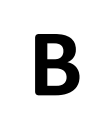

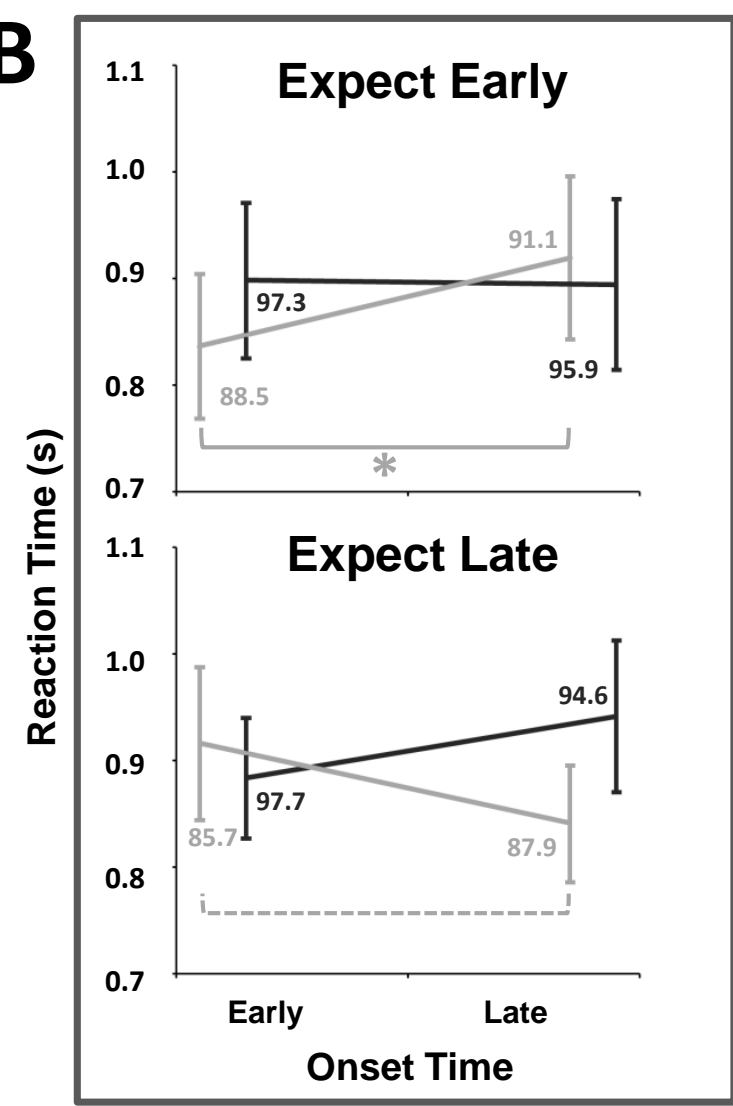

\section{Expect Late}

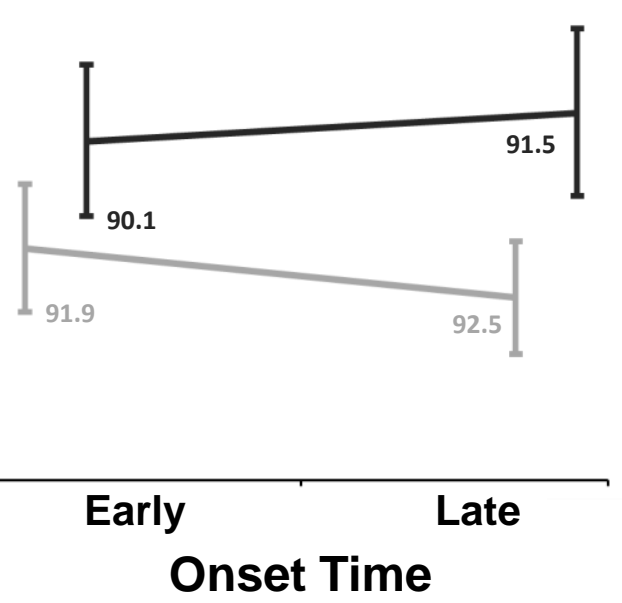

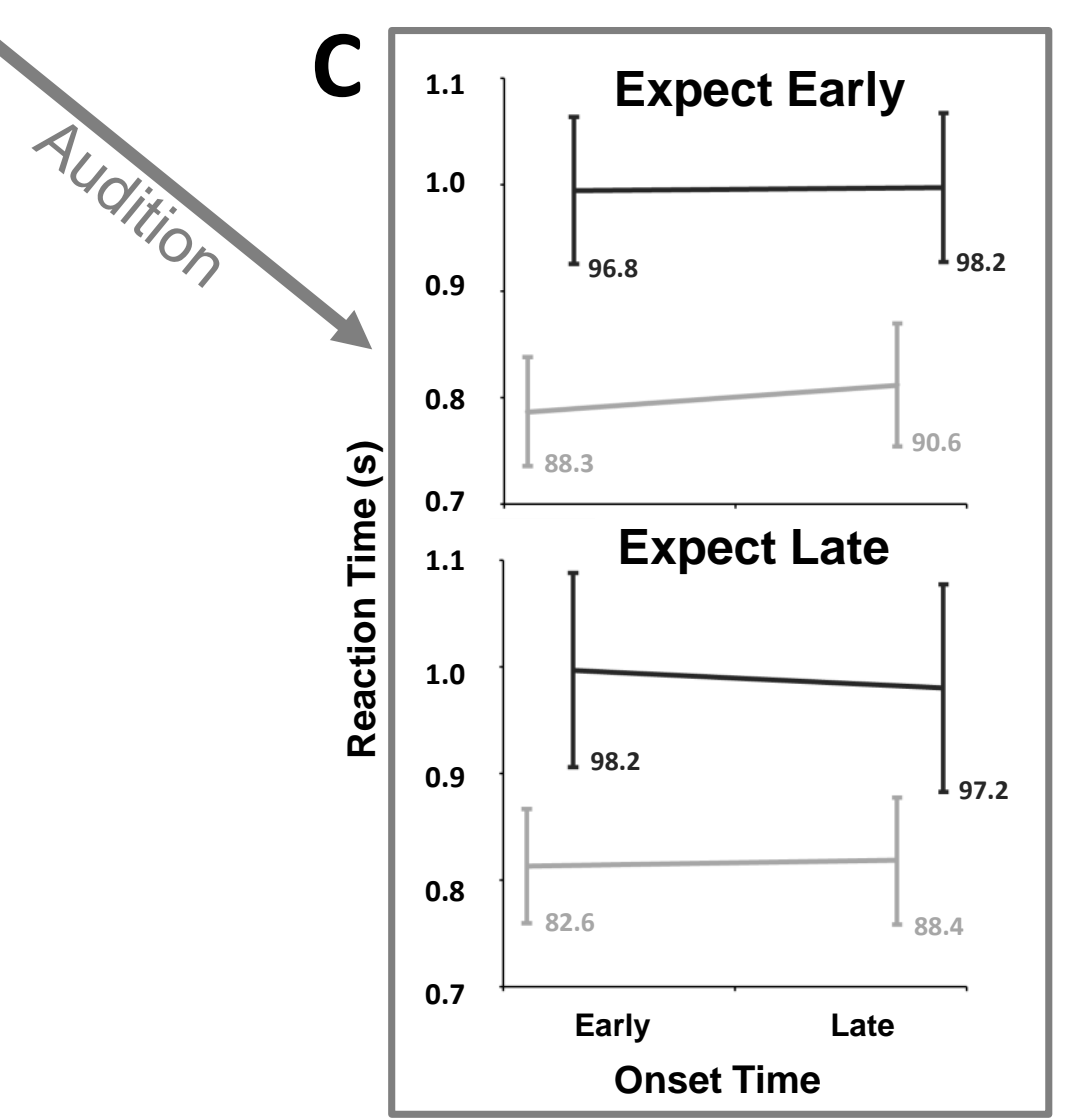



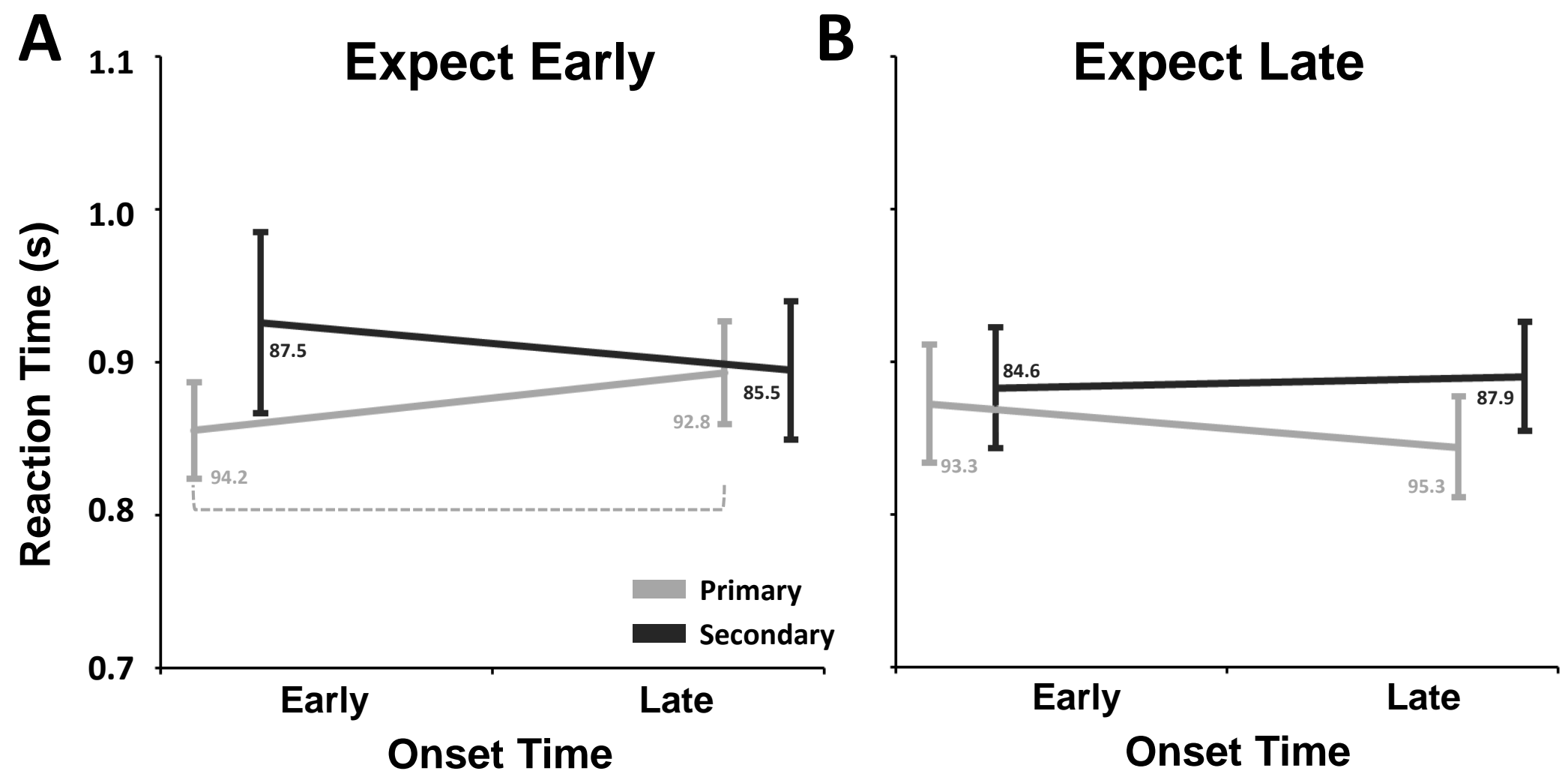